\title{
Pharmacogenetic and safety analysis of cinacalcet hydrochloride in healthy Chinese subjects
}

\author{
Yang-Jie Liu ${ }^{1 \#}$, Lu-Ning Sun ${ }^{1 "}$, Zi-Ping Cheng ${ }^{1}$, Yi Qian ${ }^{1}$, Zeng-Qing Ma ${ }^{1}$, Xue-Hui Zhang ${ }^{2}$, \\ Hong-Wen Zhang ${ }^{1}$, Li-Jun Xie ${ }^{1}$, Lei Yu ${ }^{1}$, Zi-Qing-Yun Yuan ${ }^{1}$, Yun Liu ${ }^{3}$, Yong-Qing Wang ${ }^{1,2}$ \\ ${ }^{1}$ Research Division of Clinical Pharmacology, the First Affiliated Hospital of Nanjing Medical University \& Jiangsu Province Hospital, Nanjing, \\ China; ${ }^{2}$ Department of Pharmacy, Jiangsu Shengze Hospital, Nanjing Medical University, Suzhou, China; ${ }^{3}$ Department of Geriatric Endocrinology, \\ the First Affiliated Hospital of Nanjing Medical University \& Jiangsu Province Hospital, Nanjing, China \\ Contributions: (I) Conception and design: YQ Wang; (II) Administrative support: YQ Wang, LN Sun; (III) Provision of study materials or patients: \\ HW Zhang, LJ Xie, Y Liu; (IV) Collection and assembly of data: Y Qian, ZQ Ma; (V) Data analysis and interpretation: YJ Liu, ZP Cheng; (VI) \\ Manuscript writing: All authors; (VII) Final approval of manuscript: All authors \\ "These authors contributed equally to this work. \\ Correspondence to: Yong-Qing Wang, PhD. Research Division of Clinical Pharmacology, the First Affiliated Hospital of Nanjing Medical University \\ \& Jiangsu Province Hospital, 300 Guangzhou Road, Nanjing 210009, China. Email: wyqjsph@163.com; Yun Liu, PhD. First Affiliated Hospital of \\ Nanjing Medical University \& Jiangsu Province Hospital. Email: liuyun@njmu.edu.cn.
}

Background: Our study aims to explore the effect of genetics on the pharmacodynamics (PD) and pharmacokinetics (PK) of cinacalcet in healthy Chinese subjects; to investigate the effect of dietary factors on cinacalcet, and to evaluate the safety of cinacalcet under fasting and non-fasting conditions using a bioequivalence trial.

Methods: We investigated the relationship of cinacalcet PK with single nucleotide polymorphisms (SNPs) of CYP3A4, CYP1A2 and CYP2D6, and of cinacalcet PD with SNPs of calcium-sensitive receptors (CASR) and vitamin D receptors (VDR) in 65 healthy Chinese subjects recruited to participate in this study. Our study was a phase I, open-label, randomized, two-period, two-sequence crossover, a single-center clinical study designed under both fasting and non-fasting conditions to investigate the effect of dietary factors on cinacalcet. Plasma cinacalcet concentrations were analyzed using a validated HPLC-MS/MS assay. Clinical laboratory tests evaluated safety. Thirteen SNPs of CASR, VDR, and CYP genes were selected for pharmacogenetic analysis.

Results: CYP3A4 rs4646437 was found to be associated with the PK of cinacalcet under fasting conditions $(\mathrm{P}<0.01)$. Subjects carrying $\mathrm{T}$ alleles of $\mathrm{rs} 4646437$ appeared to metabolize cinacalcet poorly. The $\mathrm{C}_{\max }$ and AUC of subjects in the non-fasting group were significantly higher $(\mathrm{P}<0.0001)$ than those in the fasting group. The $\mathrm{T}_{\max }, \mathrm{CL} / \mathrm{F}$, and $\mathrm{Vd} / \mathrm{F}$ in the fasting group were significantly higher $(\mathrm{P}<0.0001)$ than those in the non-fasting group. In the fasting group, the geometric least square mean ratios (T/R) of the $\mathrm{C}_{\max }$ and $\mathrm{AUC}_{0-\mathrm{t}}$ were $109.89 \%$ and $105.33 \%$, and the corresponding $90 \%$ CIs were $98.36-122.79 \%$ and $98.04-113.15 \%$, respectively. In the non-fasting group, the $\mathrm{T} / \mathrm{R}$ of the $\mathrm{C}_{\max }$ and $\mathrm{AUC}_{0-\mathrm{t}}$ were $100.74 \%$ and $99.09 \%$, and the corresponding 90\% CIs were $92.65-109.54 \%$ and $94.79-103.58 \%$, respectively. All adverse events (AEs) were mild, and no serious adverse events (SAEs) occurred during the bioequivalence trial.

Conclusions: Following our investigation, we reached the following conclusions: CYP3A4 rs4646437 may affect cinacalcet PK; the reference and test preparations of cinacalcet were bioequivalent under fasting and non-fasting conditions and were safe to use; and dietary factors had a significant effect on the PK of cinacalcet, in that exposure to the drug increased when cinacalcet was taken after eating.

Keywords: Cinacalcet; bioequivalence; pharmacokinetics (PK); calcium-sensitive receptors (CASR); CYP3A4

Submitted Feb 05, 2020. Accepted for publication Aug 30, 2020.

doi: $10.21037 / \mathrm{atm}-20-1329$

View this article at: http://dx.doi.org/10.21037/atm-20-1329 


\section{Introduction}

Secondary hyperparathyroidism (SHPT) is a common complication in patients with chronic renal failure $(1,2)$. Typical presenting symptoms of SHPT include elevated parathyroid hormone (PTH) levels and hyperplasia of the parathyroid glands, which can lead to bone injury, refractory pruritus, anemia, nervous system damage, and cardiovascular diseases $(3,4)$. Studies have suggested that long-term elevated levels of PTH increase the risk of death in patients with chronic kidney disease (CKD) who require dialysis $(5,6)$. Treatment of SHPT should aim to reduce parathormone concentrations through the administration of calcimimetics or active vitamin D and to stabilize calcium and phosphate metabolism (7).

Cinacalcet is the first of a new class of compounds called calcimimetics, which activates calcium-sensitive receptors (CASR) in the parathyroid gland and reduces the secretion of PTH $(8,9)$. After binding with CASR, cinacalcet enhances its sensitivity to extracellular calcium ions, which inhibits the secretion of PTH (10). These calcium-lowering effects of cinacalcet overcome the limitations of standard hypercalcemia therapy (11). In addition to inhibiting the secretion of PTH, cinacalcet can also reduce the levels of serum calcium and phosphorus in SHPT patients. Treatment with cinacalcet can also alleviate the symptoms of bone metabolism disorders and prevent vascular and soft tissue calcification, and is well-tolerated by patients, producing minimal adverse events (AEs). Cinacalcet is now used widely in the clinical treatment of SHPT patients with CKD on maintenance dialysis (7).

Additionally, some articles have shown that cinacalcet is more effective when used in patients with more severe SHPT, indicating this drug's efficacy is related to SHPT severity (12). Cinacalcet hydrochloride has been used successfully in US and Japan in the treatment of SHPT while maintaining serum levels of calcium and phosphorus (13). Cinacalcet also reduces the incidence rate of calcific uremic arteriolopathy (CUA; calciphylaxis) in patients undergoing dialysis (14). Furthermore, the usage of cinacalcet alleviates hyperparathyroidism secondary to lithium therapy in patients with the bipolar affective disorder (15).

However, cinacalcet overdose can result in hypocalcemia, and the response to cinacalcet varies significantly between individuals (16). Commonly, serum calcium decreases following the initiation of cinacalcet treatment (17); therefore, a patient's serum calcium levels should be regularly monitored while they are medicated to avoid hypocalcemia. Calcium supplementation should be considered when hypocalcemia occurs $(18,19)$. Given the high protein binding rate of cinacalcet, hemodialysis is not an effective treatment for overdose (20). Due to the significant variation in individual response to this drug, it is necessary to develop individual treatment plans for patients taking cinacalcet.

The elimination of cinacalcet is principally mediated by oxidative metabolism, partly by cytochrome P450 (CYP) $3 \mathrm{~A} 4$ (21). It has been reported that cinacalcet is metabolized primarily by CYP3A4, CYP2D6, and CYP1A2, of which CYP3A4 is the dominant metabolic enzyme (7). As cinacalcet is also a strong inhibitor of CYP2D6 (22), when it is co-administered with drugs metabolized by CYP2D6 with narrow therapeutic targets, the dosage of cinacalcet may need adjustment (23). However, there is no literature describing the effect of single nucleotide polymorphisms (SNPs) of CYP enzymes on the pharmacokinetics (PK) of cinacalcet. Reports on the effect of SNPs are limited to CASR, which acts as the interaction site of cinacalcet and other calcium regulatory receptors, including vitamin D receptors (VDR) (16,24-27).

This study was conducted to investigate the effect of dietary factors on cinacalcet and to evaluate the safety of this drug in a bioequivalence trial using two types of cinacalcet hydrochloride tablets. Further, SNPs of CASR and VDR have been reported to affect cinacalcet pharmacodynamics (PD). In the study, we investigated whether these SNPs had any impact on cinacalcet response in healthy Chinese subjects after receiving a single dose under fasting conditions. We selected SNPs of CYP3A4, CYP2D6, and CYP1A2 enzymes, which are commonly reported to affect the metabolism of other drugs, to explore whether they have any effect on the PK of cinacalcet under fasting conditions (28-32). We present the following article in accordance with the CONSORT reporting checklist (available at http://dx.doi.org/10.21037/atm-20-1329).

\section{Methods}

\section{Subjects}

One hundred thirty-four healthy subjects aged 18-65 years (inclusive) with a body mass index (BMI) in the range of $19-26 \mathrm{~kg} / \mathrm{m}^{2}$ (inclusive) were enrolled in this study. Subjects that had clinically significant abnormalities in electrocardiogram results, blood chemistry, or urinalysis were excluded from this study, as were those who returned 
Table 1 Demographic characteristics

\begin{tabular}{lll}
\hline Characteristics & Fasting group $(\mathrm{n}=65)$ & Fed group $(\mathrm{n}=63)$ \\
\hline Age (years), mean \pm SD (range) & $25.4 \pm 4.2(18$ to 40$)$ & $25.5 \pm 4.6(18$ to 39$)$ \\
Sex, $\mathrm{n}(\%)$ & & \\
$\quad$ Male & $49(71.0)$ & $45(70.3)$ \\
Female & $20(29.0)$ & $19(29.7)$ \\
Height $(\mathrm{cm})$, mean $\pm \mathrm{SD}$ (range) & $169.15 \pm 7.92(152.9$ to 186.8$)$ & $170.11 \pm 8.13(149.3$ to 188.1$)$ \\
Weight $(\mathrm{kg})$, mean $\pm \mathrm{SD}$ (range) & $65.03 \pm 6.62(50.3$ to 80.8$)$ & $64.01 \pm 7.13(49.3$ to 82.1$)$ \\
BMI $\left(\mathrm{kg} / \mathrm{m}^{2}\right)$, mean $\pm \mathrm{SD}$ (range) & $22.74 \pm 1.89(19.2$ to 25.9$)$ & $22.07 \pm 1.77(19$ to 26$)$ \\
\hline
\end{tabular}

positive pregnancy tests. None of the subjects consumed excessive alcohol or smoked, and none had taken any drugs for at least two weeks before the study. Baseline hematology and clinical biochemistry laboratory parameters were within normal limits for all participants. After receiving oral and written explanations, subjects gave written informed consent before the commencement of the study.

In the fasting group, of the 69 enrolled, 65 subjects completed the study. Four withdrew due to AEs after the first cycle of administration. In the non-fasting group, of the 65 subjects enrolled, two withdrew, and 63 completed the study. One subject withdrew on his initiative before drug administration, and another was found not to match their given identity. The demographic characteristics of the study participants are shown in Table 1 .

\section{Study design}

This study (China Food and Drug Administration registration: 2016L07520; Drug clinical trial registration number: CTR20160747; full trial protocol (including dates defining the periods of recruitment and follow-up): http:// www.chinadrugtrials.org.cn/clinicaltrials.searchlistdetail. dhtml) was a phase I, open-label, randomized, two-period, two-sequence crossover, single-center clinical trial designed to evaluate the bioequivalence of cinacalcet hydrochloride $25 \mathrm{mg}$ tablets The study was conducted in accordance with the Declaration of Helsinki (as revised in 2013). The study was approved by ethics board of the First Affiliated Hospital of Nanjing Medical University (No. 2016-MD-208) and informed consent was taken from all the patients. Each subject participated in two phases, a test administration and a reference administration phase (Figure 1), under fasting or non-fasting conditions (33). The test preparation was provided by Jiangsu Jiayi Medicine Co.,
Ltd., and the reference preparation, REGPARA, came from Kyowa Hakko Kirin Co., Ltd. Each subject underwent two cycles of administration, randomized to receive the test preparation $(\mathrm{T})$ or the reference preparation $(\mathrm{R})$ at a dose of $50 \mathrm{mg}$. The random table (including random allocation sequence) was generated by the statistical analysis company using SAS software Proc Plan process. Block randomization method was used to randomize. The order of each subject receiving the test preparation or reference preparation was determined by the random table after the sponsor completed the recruitment of the subjects. The intervening period between the two cycles was not less than 10 days. For the non-fasting group, test and reference preparations were administered 30 minutes after high-fat, highcalorie breakfast with the recommendations of regulatory guidelines.

This study didn't include any interim analyses. The trial would be stopped if the following conditions occur: (I) major mistakes in the clinical trial protocol were found in the trial, making it difficult to evaluate the drug; (II) the sponsor requested suspension under the premise of fully protecting the rights and safety of subjects; (III) the State Drug Administration or the ethics committee ordered the suspension of the trial for some reason; (IV) the clinical trial cannot be continued due to other reasons.

\section{Bioanalytical assay}

A confirmatory method was developed with highperformance liquid chromatography-tandem mass spectrometry (HPLC-MS/MS) to detect the concentration of cinacalcet in plasma. Cinacalcet hydrochloride- $\mathrm{d}_{3}$ was used as an internal standard. The analyte and internal standard were extracted by protein precipitation and separated from the matrix by a reversed-phase 


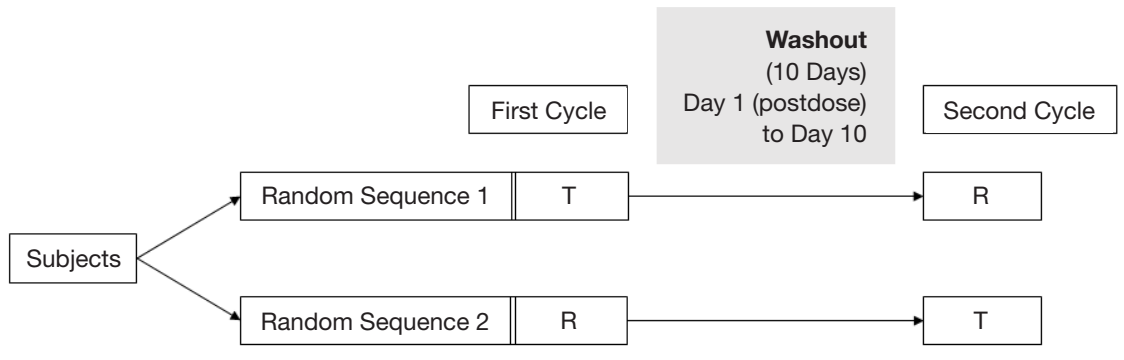

\begin{tabular}{|l|l|l|}
\hline T: Test formulation, \\
Cinacalcet hydrochloride tablet $25 \mathrm{mg} \times 2$ \\
(Jiangsu Jiayi Medicine Co., Ltd.) \\
For 1 single dose
\end{tabular}

Figure 1 Schematic illustration of study design. $4 \mathrm{~mL}$ of peripheral blood was collected via an indwelling cannula into tubes holding the anticoagulant K2-ethylenediaminetetraacetic acid. During each treatment period, samples were collected at the following time points: 0 , 0.25 , $0.5,1,1.5,2,2.5,3,3.5,4,5,6,8,12,24,48,72$ and $96 \mathrm{~h}$ after each single-dose administration. After collection, the blood samples were centrifuged within $30 \mathrm{~min}$ at $4{ }^{\circ} \mathrm{C}$ at $3,000 \times \mathrm{g}$ for $8 \mathrm{~min}$, and the supernatant plasma samples were stored at $-70{ }^{\circ} \mathrm{C}$ until further analysis.

chromatographic column. A tandem quadrupole mass spectrometer with electrospray ionization mode (ESI) was used to conduct the quantitative analysis. The dynamic range of the assay was $0.100-30.0 \mathrm{ng} / \mathrm{mL}$. The intraassay precision and accuracy were less than $8.0 \%$ and 97.3-105.0\%, respectively. The inter-assay precision and accuracy were less than $5.8 \%$ and $97.7-103.0 \%$, respectively.

This trial was an open trial. Except for biological sample analysts, other personnel such as clinical researchers, participants, project management personnel, project supervisors, statistics and data management analysts were not blinded, only sample analysis personnel used blind analysis.

\section{Safety and pharmacodynamic parameter evaluations}

AEs evaluated safety, vital signs, physical examination, clinical laboratory tests (complete blood count, blood biochemistry, and urinalysis), coagulation check, and 12lead electrocardiogram. The severity of all AEs and serious adverse events (SAEs) were assessed by clinicians and their potential relationship to medications.

To assess plasma intact PTH (iPTH) and serum calcium concentrations, blood samples were collected before dosing and at the fourth and 96th hour after drug administration. iPTH and serum calcium levels were measured by a fully automatic biochemistry analyzer (Beckman Coulter, AU5800).

\section{Statistical analysis}

Standard non-compartmental PK parameters $\left(\mathrm{C}_{\max }, \mathrm{T}_{\max }\right.$, $t_{1 / 2}, A C_{0-t}, A C_{0-\infty}$, etc) were calculated by WinNonlin 7.0. Bioequivalence was assessed separately in the fasting and non-fasting groups. A $90 \% \mathrm{CI}$ of the ratio of geometric means in the range of $80.00-125.00 \%$ between the test and reference preparations would be considered bioequivalent. SAS 9.4 was used for the statistical analyses. Statistically significant differences were determined by one-way analysis of variance (ANOVA) with an unpaired two-tailed heteroscedastic $t$-test. Statistical significance was set at $\mathrm{P}<0.05$.

\section{Genotype selection and analysis}

Blood samples were obtained from the 65 fasting group subjects before the administration of cinacalcet for genotyping purposes. DNA was extracted from each subject's leukocytes using a commercially available kit (Relax Gene Blood DNA System; TIANGEN BIOTECH (BEIJING), China) (34). DNA samples were sent to Sangon Biotech for sequencing with a DNA sequencer (ABI3730, AB Sciex). Thirteen SNPs in five genes were selected for sequencing. Of these genes, CASR and $V D R$ are involved in PTH and calcium regulation (16), and CYP3A4, CYP2D6, and CYP1A2 are known as the major enzymes involved in cinacalcet hydrochloride metabolism (7). Table 2 sets out information on the 13 selected SNPs in the CASR, VDR, 
Table 2 The information of 13 SNPs in CASR, VDR, CYP3A4, CYP2D6 and CYP1A2 genes in Chinese healthy subjects (n=65)

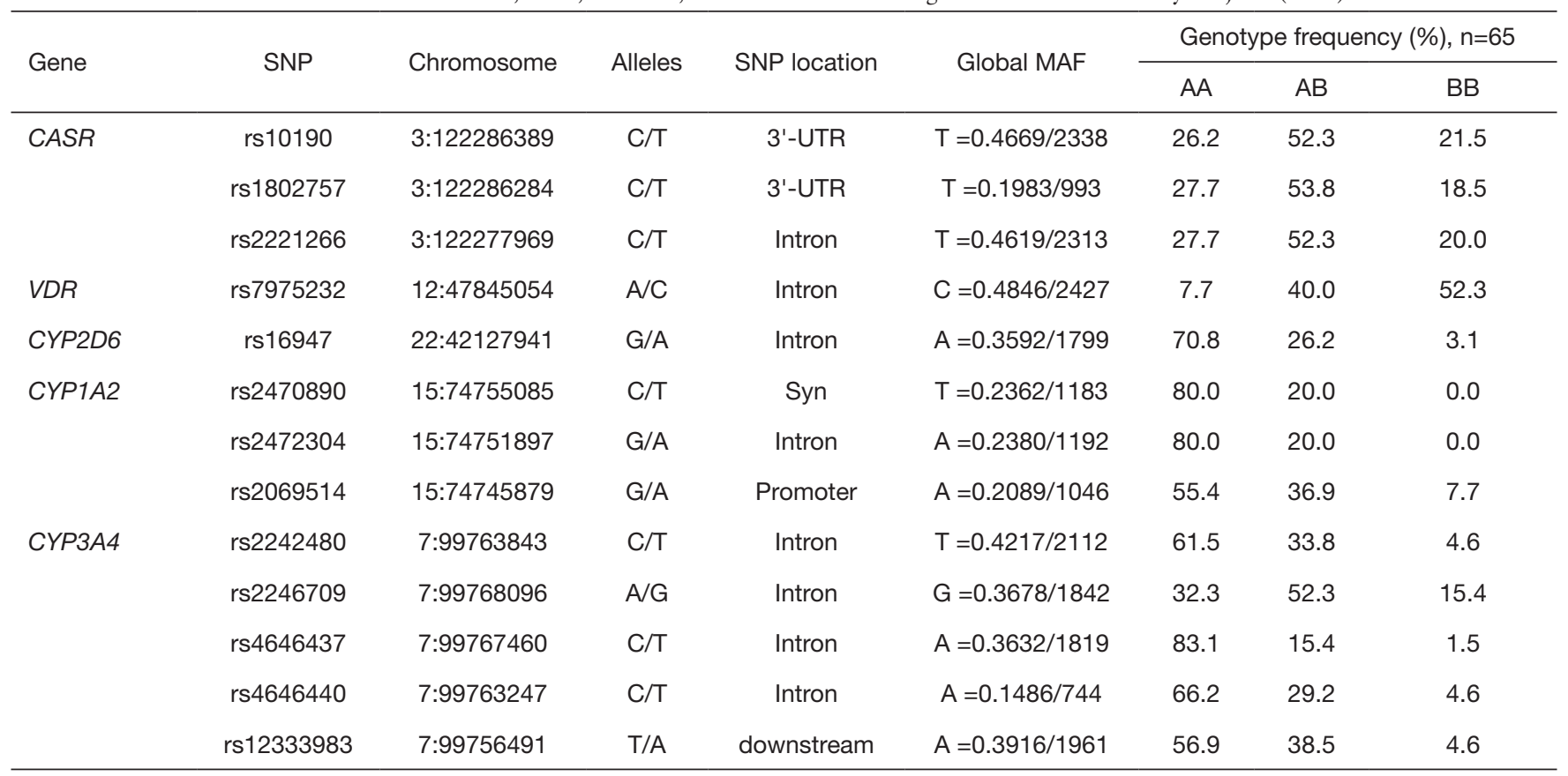

AA, homozygote major allele; $A B$, heterozygote allele; BB, homozygote minor allele; SNP, single nucleotide polymorphism.

CYP3A4, CYP2D6, and CYP1A2 genes in the 65 healthy subjects.

\section{Results}

\section{Safety and tolerability}

In the fasting group, a total of 84 AEs occurred in 42 subjects, including 50 after receiving the test preparation and 34 after receiving the reference preparation. Four subjects withdrew due to AEs after the first cycle of administration. One of these subjects exhibited prolonged electrocardiogram (ECG) QRS wave duration, indicating an intraventricular block; two subjects had low fibrinogen, and one subject displayed abnormal coagulation function. Clinicians believed it was not appropriate for these subjects to continue with the experiment. In the non-fasting group, 71 AEs occurred in 31 subjects, including 45 after receiving the test preparation and 26 after receiving the reference preparation. One subject vomited after receiving the test preparation in the first cycle, and one subject vomited after receiving the test preparation in the second cycle. All AEs were mild, and no SAEs occurred in this study. These results show that the test and reference preparations were both safe under fasting and non-fasting conditions. Table 3 sets out the specific symptoms of AEs during preparation administration.

\section{$P K$ and bioequivalence}

The mean cinacalcet plasma concentration-time profiles for the test and reference preparations under fasting and non-fasting conditions are presented in Figure 2. The corresponding PK parameters are summarized in Table 4 and Figure 3.

Statistical comparisons of cinacalcet hydrochloride between the test and reference preparations demonstrated that the geometric least square mean ratios $(T / R)$ of $C_{\max }$, $\mathrm{AUC}_{0-\mathrm{t}}$, and $\mathrm{AUC}_{0-\infty}$ values in the fasting group were $109.89 \%, 105.33 \%$, and $108.83 \%$ respectively, and the corresponding 90\% CIs were 98.36-122.79\%, 98.04$113.15 \%$, and $98.90-119.75 \%$. In the non-fasting group, the $\mathrm{T} / \mathrm{R}$ of $\mathrm{C}_{\max }, \mathrm{AUC}_{0-\mathrm{t}}$, and $\mathrm{AUC}_{0-\infty}$ values were $100.74 \%$, $99.09 \%$ and $100.26 \%$ respectively, and the corresponding $90 \%$ CIs were $92.65-109.54 \%, 94.79-103.58 \%$, and 96.00-104.71\%. All CIs fell within the conventional 80.00-125.00\% acceptance limits for bioequivalence (Table 5). There was no significant difference in $T_{\max }$ found between the two treatments under either fasting or nonfasting conditions (33). Additionally, the $\mathrm{C}_{\max }$ and $\mathrm{AUC}$ of 
Table 3 Specific symptoms of AEs during administration

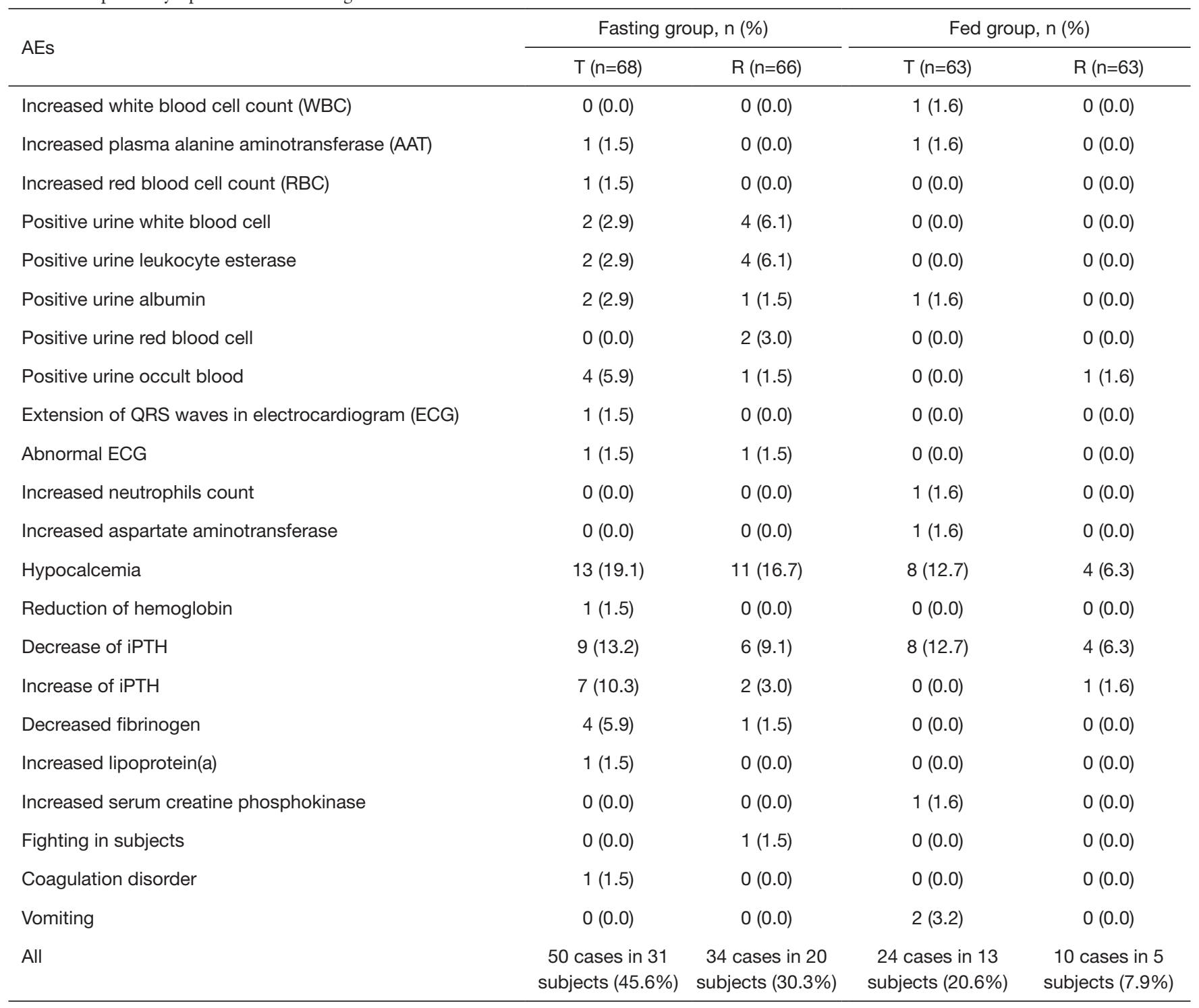

subjects in the non-fasting group were significantly higher $(\mathrm{P}<0.0001)$ than those in the fasting group. The $\mathrm{T}_{\max }$, $\mathrm{CL} / \mathrm{F}$, and $\mathrm{Vd} / \mathrm{F}$ in the fasting group were significantly higher $(\mathrm{P}<0.0001)$ than those in the non-fasting group. No significant difference in $t_{1 / 2}$ and $\lambda_{z}$ was found between the two groups.

\section{PD of cinacalcet in healthy subjects}

Changes in $\mathrm{PPTH}$ and serum calcium concentrations during the study are shown in Figure 4. These changing values varied widely between the subjects. iPTH and serum calcium declined significantly from the baseline at four hours post-administration. Ninety-six hours postadministration, $\mathrm{iPTH}$ and serum calcium returned to the baseline in both the fasting and non-fasting group. The specific concentrations of iPTH and serum calcium at different points in time are shown in Table 6.

\section{Association of CASR and VDR genotypes with PD of cinacalcet in the fasting group}

No significant differences were found between SNPs of VDR and CASR concerning the changing level of iPTH 

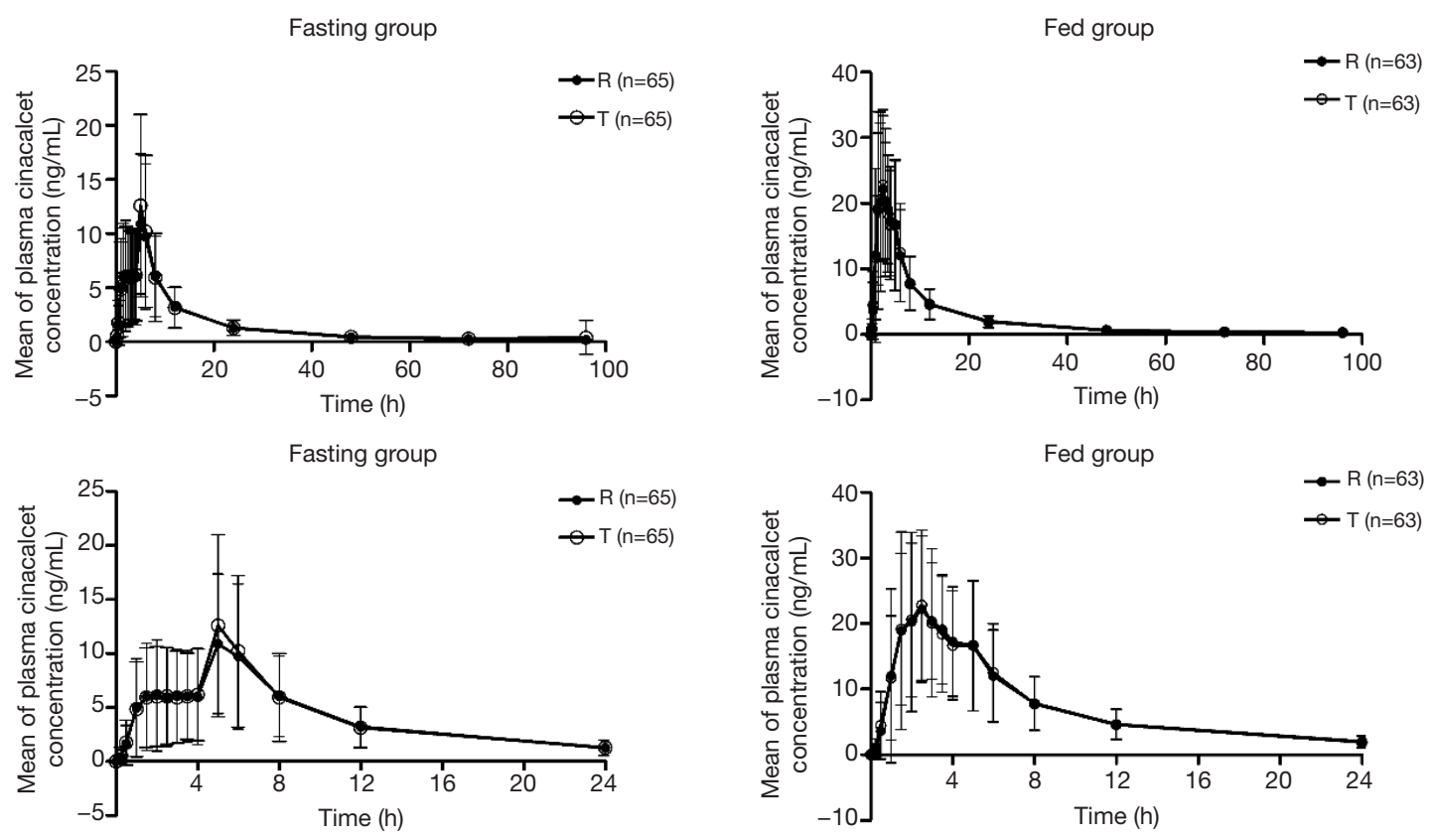

Figure 2 Arithmetic mean (SD) cinacalcet plasma concentration-time profiles for the test and reference preparations in the fasting and nonfasting groups. Dose: $50 \mathrm{mg}$; R: reference administration; T: test administration.

Table 4 Summary of pharmacokinetic parameters of cinacalcet following administration of a single oral dose $(50 \mathrm{mg})$ of the test and reference preparations in healthy Chinese subjects (mean $\pm \mathrm{SD}$ )

\begin{tabular}{|c|c|c|c|c|}
\hline PK parameters of cinacalcet & \multicolumn{2}{|c|}{ Fasting group $(n=65)$} & \multicolumn{2}{|c|}{ Fed group $(n=63)$} \\
\hline $\mathrm{AUC}_{0-\mathrm{t}}(\mathrm{ng} \cdot \mathrm{h} / \mathrm{mL})$ & $138.9 \pm 73.0$ & $134.1 \pm 70.09$ & $230.0 \pm 99.34$ & $231.5 \pm 100.2$ \\
\hline $\mathrm{AUC}_{0-\infty}(\mathrm{ng} \cdot \mathrm{h} / \mathrm{mL})$ & $150.5 \pm 80.11$ & $145.3 \pm 75.88$ & $248.6 \pm 108.3$ & $247.9 \pm 109.2$ \\
\hline $\mathrm{C}_{\max }(\mathrm{ng} / \mathrm{mL})$ & $14.26 \pm 8.176$ & $13.20 \pm 7.149$ & $28.08 \pm 12.73$ & $28.24 \pm 15.16$ \\
\hline$t_{1 / 2}(h)$ & $37.42 \pm 20.47$ & $34.34 \pm 15.68$ & $39.46 \pm 20.56$ & $35.98 \pm 12.95$ \\
\hline$\lambda_{z}(1 / h)$ & $0.024 \pm 0.013$ & $0.026 \pm 0.016$ & $0.023 \pm 0.012$ & $0.022 \pm 0.009$ \\
\hline $\mathrm{CL} / \mathrm{F}(\mathrm{L} / \mathrm{h})$ & $436.9 \pm 268.3$ & $439.2 \pm 265.2$ & $251.0 \pm 159.7$ & $251.7 \pm 164.4$ \\
\hline $\mathrm{Vd} / \mathrm{F}(\mathrm{L})$ & $21,099 \pm 13,983$ & $20,595 \pm 12,205$ & $13,208 \pm 8,071$ & $11,883 \pm 5,257$ \\
\hline
\end{tabular}

in 65 healthy subjects after receiving test or reference cinacalcet tablets under fasting conditions (Figure 5). For VDR rs7975232, the mean changing level of iPTH was lower in subjects with AA genotype (homozygote minor allele) than those with AC (heterozygote allele) and CC (homozygote major allele) genotypes. CASR rs10190, rs1802757, and rs2221266 showed a similar trend. However, no statistical differences were found due to the volatile nature of data between individuals.

\section{Association of CYPs genotypes with PK of cinacalcet in the} fasting group

A significant difference was found between the SNP of CYP3A4 rs4646437 and the PK parameters $\left(\mathrm{C}_{\max }\right.$ and $\left.\mathrm{AUC}_{0-\mathrm{t}}\right)$ in 65 healthy subjects after receiving test or reference cinacalcet tablets under fasting conditions (Figure 6). The average $\mathrm{C}_{\max }$ and $\mathrm{AUC}_{0-\mathrm{t}}$ of subjects carrying $\mathrm{T}$ alleles of rs 4646437 were higher than those with major 

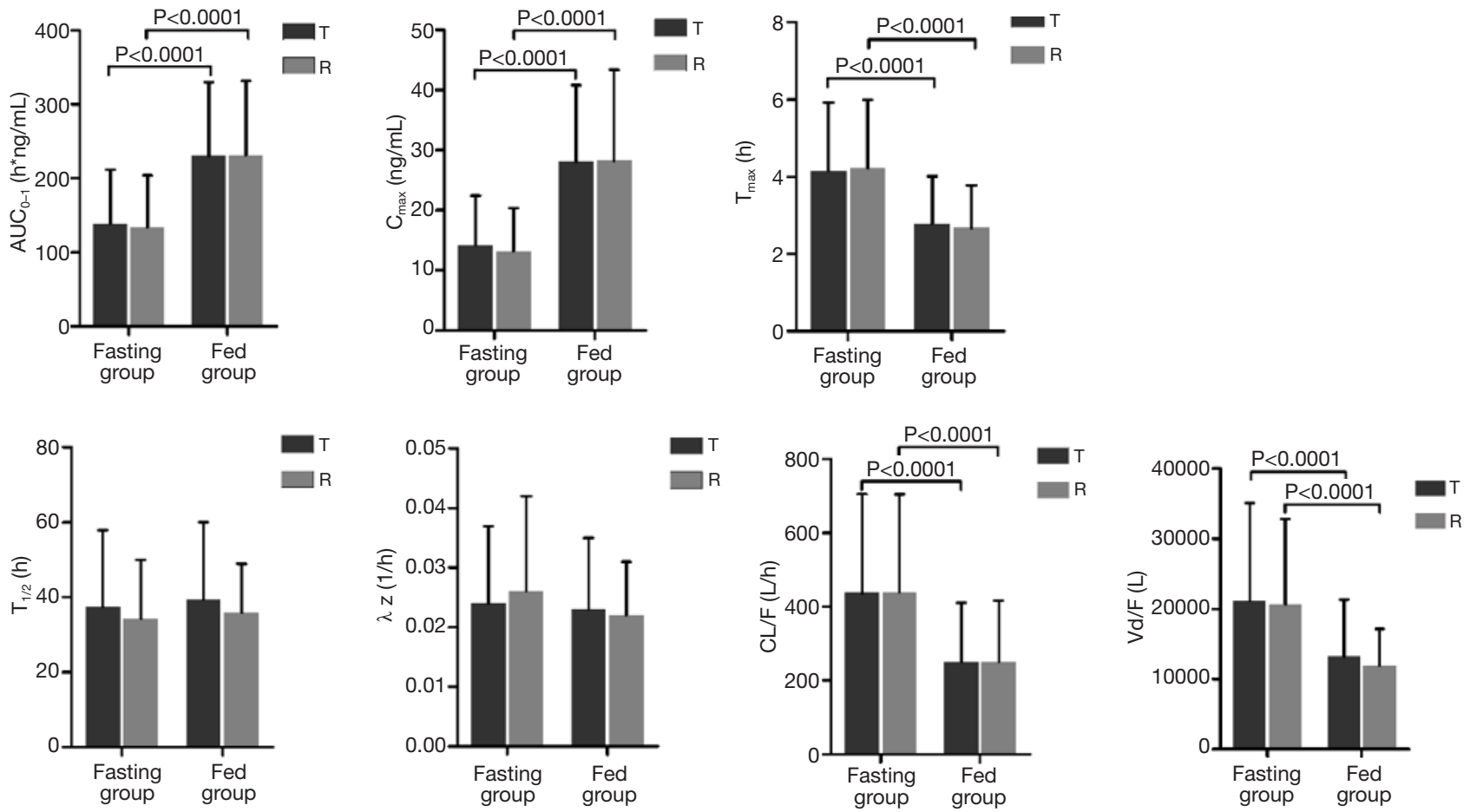

Figure 3 Comparison diagram of PK parameters of cinacalcet between fasting and non-fasting group. Dose: $50 \mathrm{mg}$; R: reference administration; T: test administration. PK, pharmacokinetics.

Table 5 Statistical comparison of pharmacokinetic parameters for cinacalcet following administration of a single oral dose of the test and reference preparations under both fasting and fed conditions in healthy Chinese subjects

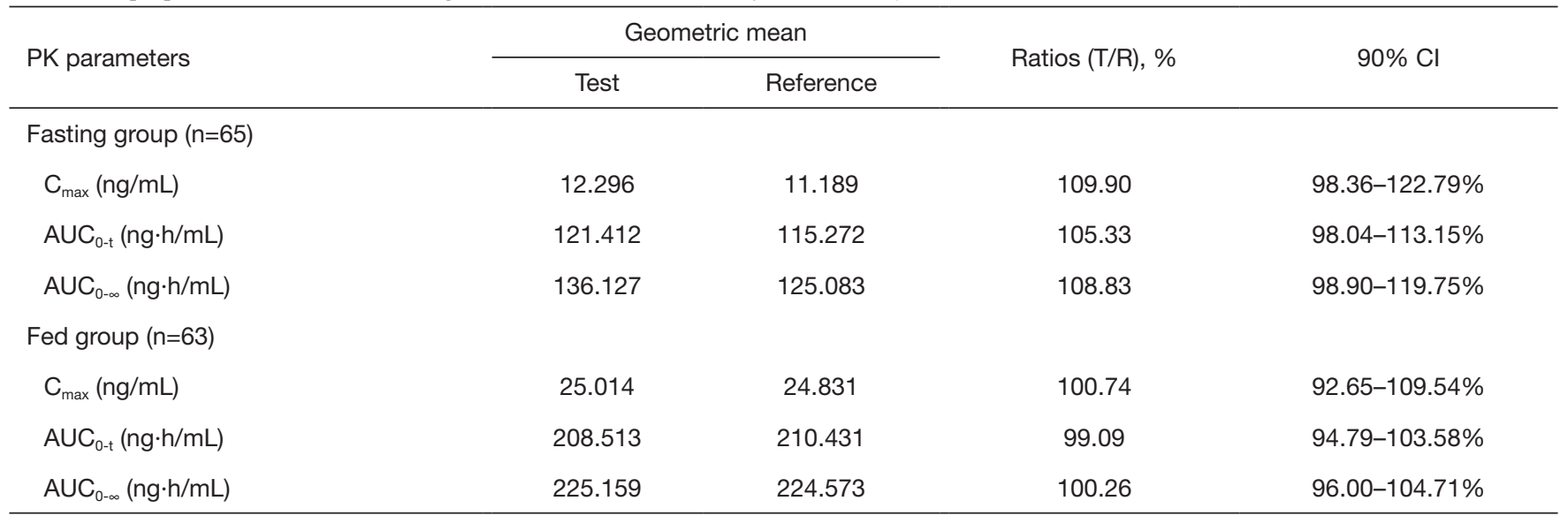

homozygote alleles (CC).

\section{Discussion}

This clinical trial was conducted to compare the bioequivalence between test and reference preparations of cinacalcet hydrochloride tablets and to evaluate the safety of cinacalcet. The results demonstrated that the reference and test preparations were bioequivalent under both fasting and non-fasting conditions. Safety evaluations revealed that both the test and reference preparations were well tolerated by subjects and were safe to use.

Common AEs of cinacalcet observed in dialysis patients include hypocalcemia, nausea, vomiting, diarrhea, 

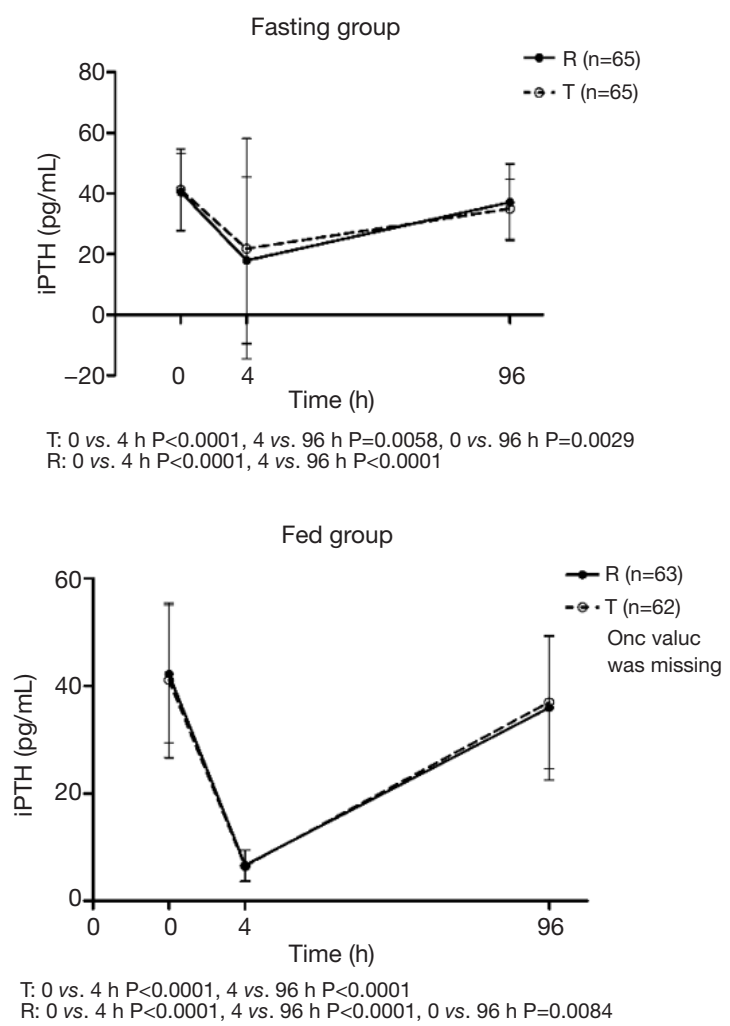
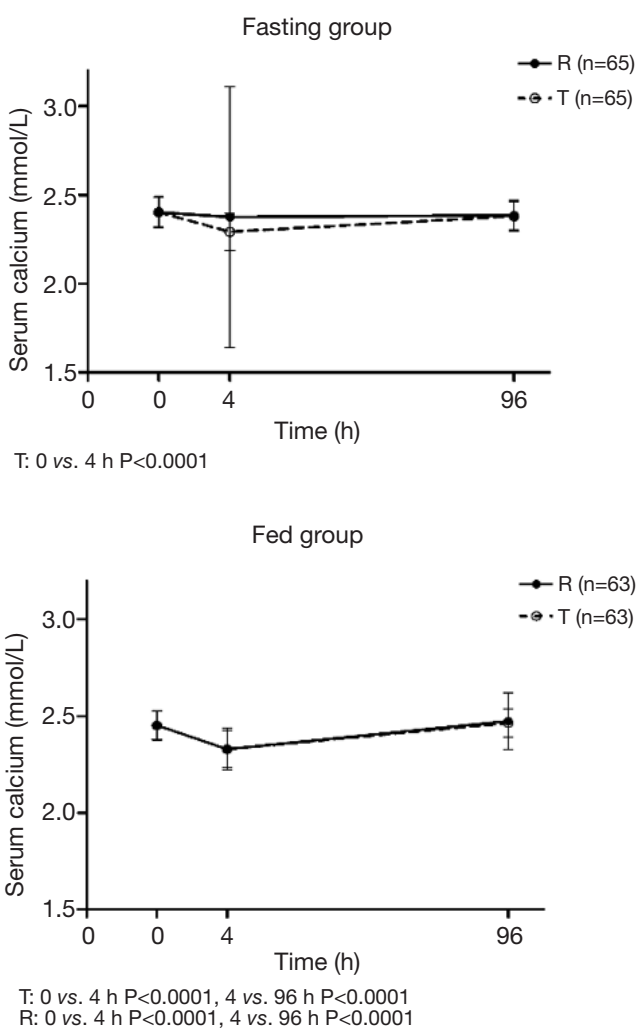

Figure 4 Mean (SD) change of iPTH and serum calcium concentration in the fasting and non-fasting groups after receiving test and reference administration. Dose: $50 \mathrm{mg}$; R: reference administration; T: test administration.

Table 6 Pharmacodynamic profiles for cinacalcet following administration of a single oral dose $(50 \mathrm{mg})$ of the test and reference preparations under both fasting and fed conditions in healthy Chinese subjects (mean \pm SD)

\begin{tabular}{|c|c|c|c|c|}
\hline PD profiles of cinacalcet & \multicolumn{2}{|c|}{ Fasting group $(n=65)$} & \multicolumn{2}{|c|}{ Fed group $(n=63)$} \\
\hline \multicolumn{5}{|l|}{ iPTH (pg/mL) } \\
\hline $\mathrm{Oh}$ & $41.21 \pm 13.44$ & $40.54 \pm 12.72$ & $41.00 \pm 14.34$ & $42.23 \pm 12.89$ \\
\hline $4 \mathrm{~h}$ & $21.81 \pm 36.39$ & $17.95 \pm 27.39$ & $6.520 \pm 2.926$ & $6.670 \pm 2.784$ \\
\hline \multicolumn{5}{|l|}{ Serum calcium (mmol/L) } \\
\hline $\mathrm{Oh}$ & $2.401 \pm 0.08400$ & $2.402 \pm 0.08605$ & $2.452 \pm 0.07576$ & $2.453 \pm 0.7265$ \\
\hline $4 \mathrm{~h}$ & $2.291 \pm 0.1035$ & $2.375 \pm 0.7356$ & $2.329 \pm 0.1083$ & $2.329 \pm 0.09498$ \\
\hline $96 \mathrm{~h}$ & $2.379 \pm 0.08427$ & $2.385 \pm 0.08290$ & $2.464 \pm 0.07482$ & $2.475 \pm 0.1474$ \\
\hline
\end{tabular}

abdominal distention, myalgia, dizziness, hypertension, asthenia, anorexia, prolongation of QT interval, and noncardiac chest pain $(7,35,36)$. There are limited reports of cinacalcet AEs occurring in healthy subjects. The most frequently occurring $\mathrm{AE}$ observed in our study was hypocalcemia, followed by changes in plasma iPTH $(37,38)$. Reports describing the prognosis of patients administered cinacalcet are currently lacking (39). Additionally, all AEs 

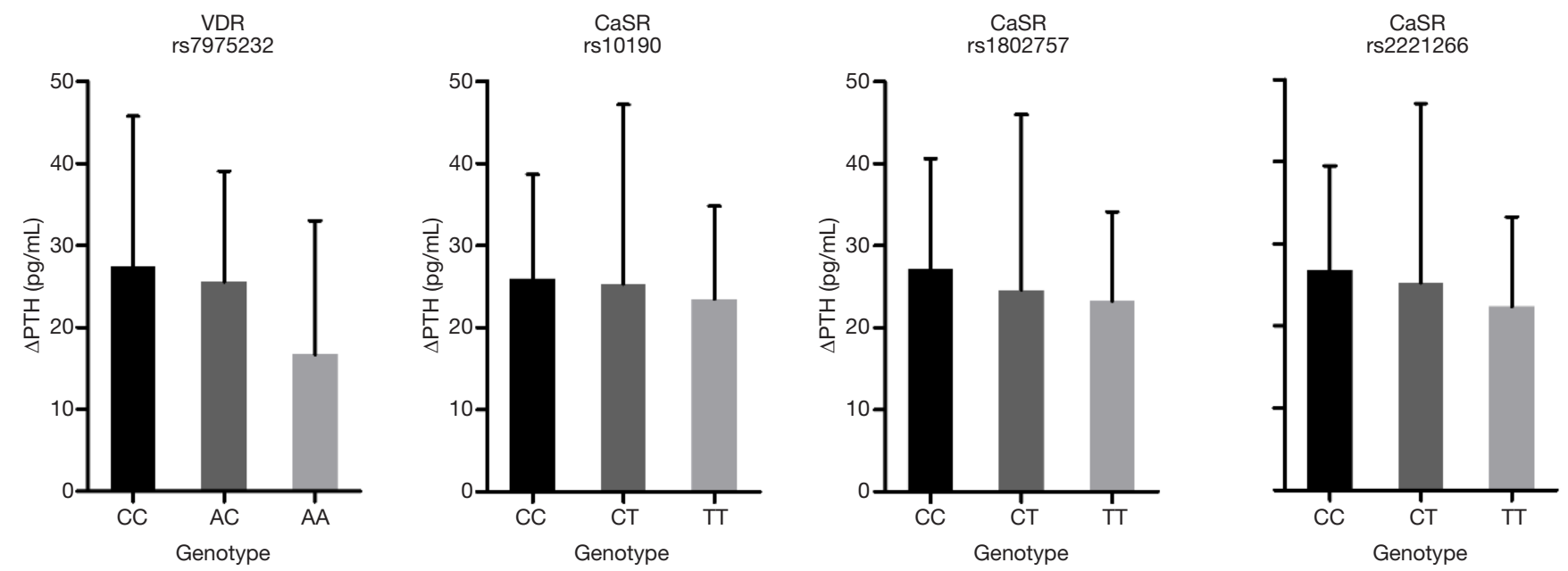

Figure 5 No statistically significant associations were found between SNPs of VDR and CASR and the changing level of iPTH in 65 healthy subjects after receiving $50 \mathrm{mg}$ of cinacalcet under fasting conditions. $\Delta \mathrm{PTH}=\mathrm{iPTH}_{\mathrm{Baseline}}-\mathrm{iPTH}_{4 \mathrm{~h}}(\mathrm{pg} / \mathrm{mL})$; $\Delta \mathrm{Ca}=\mathrm{Ca}_{\text {Baseline }}-\mathrm{Ca}_{4 \mathrm{~h}}(\mathrm{mmol} / \mathrm{L})$, the values were obtained by calculating the average of two cycles after subjects receiving test and reference administration).
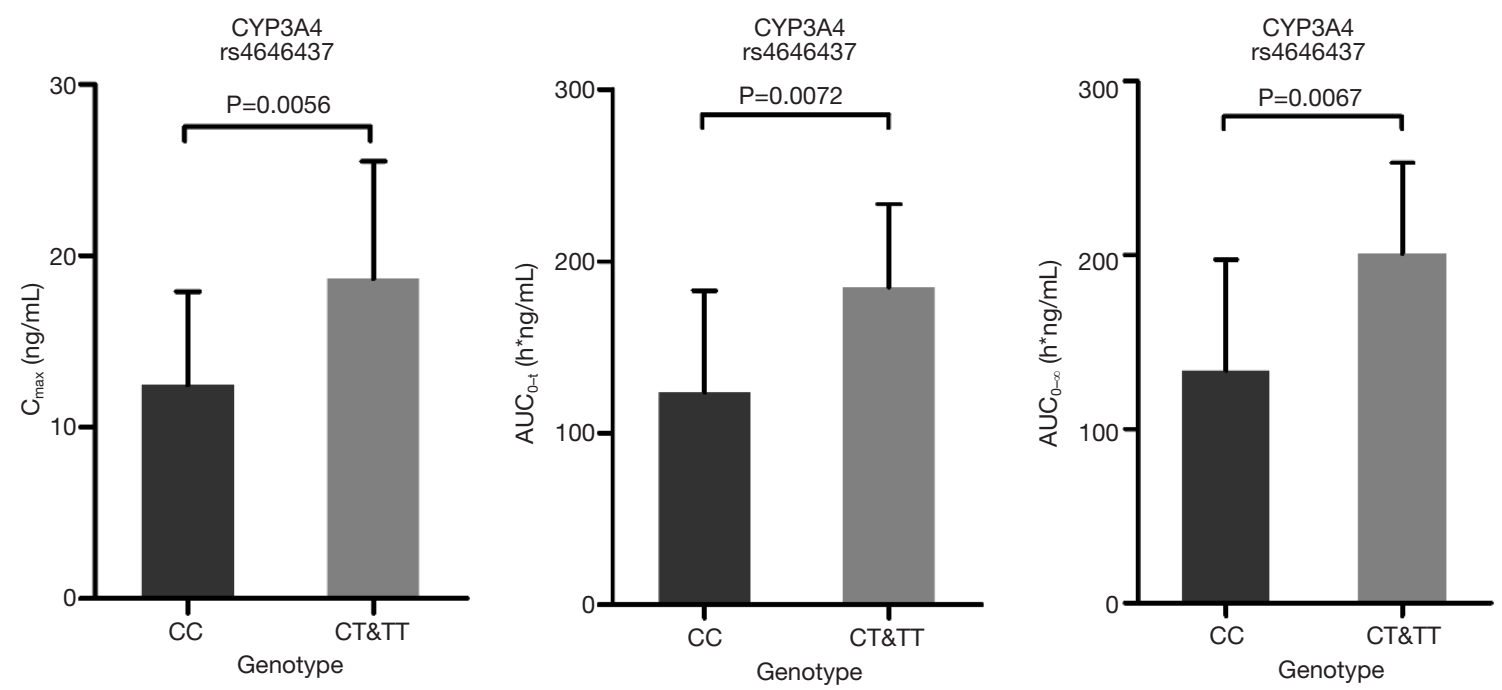

Figure 6 Significant associations were found between CYP3A4 rs4646437 and $\mathrm{C}_{\max }$ and AUC in 65 healthy subjects after receiving 50 mg of cinacalcet under fasting conditions. The values were obtained by calculating the average of two cycles after subjects receiving test and reference administration. Because only one subject was with TT genotype, so the subjects with CT and TT genotypes were classified as the same group for statistics.

with an incidence of more than $5 \%$ occurred in the fasting group, including decreased fibrinogen (5.9\%), occult blood in the urine $(5.9 \%)$, white blood cells in the urine $(6.1 \%)$ and leukocyte esterase in the urine (6.1\%). All AEs were mild, and no SAEs occurred, which shows both the test and reference cinacalcet tablets were well tolerated and safe.
Changes in iPTH and serum calcium differed significantly among subjects after receiving either the test or reference preparations under fasting conditions, while subjects in the non-fasting group showed fewer individual differences. Also, the $\mathrm{C}_{\max }$ and AUC of subjects in the nonfasting group were significantly higher $(\mathrm{P}<0.0001)$ than in 
the fasting group, suggesting that dietary factors affected the PK and PD of cinacalcet. Taking cinacalcet with meals or immediately after eating may improve its absorption.

It has been reported that CASR rs1802757 and rs10190 are significantly associated with individual variation in cinacalcet response (16). Studies have also previously found that CASR rs2221266 may be associated with differences in PTH levels and that the VDR gene polymorphism rs7975232 is associated with $\mathrm{PTH}$ and calcium regulation $(25,26)$. However, the current study found no significant associations between SNPs of VDR and CASR and changes in $\mathrm{PPTH}$ and calcium levels (34). A study by Jeong et al. recruited SHPT patients who had been taking cinacalcet for more than three months, and patients whose iPTH levels were elevated even after three months of cinacalcet treatment were defined as non-responders. However, all subjects in the current study were healthy, and each participant exhibited a decline in iPTH at least once after a single dose. Therefore, diverse factors may affect the significant associations between SNPs and cinacalcet PD, and SNPs of CASR and VDR may influence cinacalcet PD less under non-pathological conditions.

CYP3A4 is the dominant enzyme that metabolizes cinacalcet, others being CYP1A2 and CYP2D6 $(7,16)$. No literature has yet reported whether these CYP enzymes affect the PK of cinacalcet (40). In the current study, we found that the $\mathrm{C}_{\max }$ and AUC of subjects with T alleles of CYP3A4 rs4646437 were significantly higher than those with the CC genotype $(\mathrm{P}<0.01)$, indicating that the SNP of CYP3A4 rs4646437 may affect cinacalcet PK under fasting conditions.

\section{Conclusions}

CYP3A4 rs4646437 may influence the PK of cinacalcet. Significant associations were found in the current study between CYP3A4 rs4646437 and PK parameters $\left(\mathrm{C}_{\max }\right.$ and AUC) of cinacalcet $(\mathrm{P}<0.01)$. Subjects carrying $\mathrm{T}$ alleles of rs4646437 appeared to metabolize cinacalcet poorly, and the reference and the test cinacalcet preparations were found to be bioequivalent in healthy Chinese subjects under both fasting and non-fasting conditions. An evaluation of drug safety showed that cinacalcet was well-tolerated by patients and that it was safe to use. Also, it was found that dietary factors had a significant effect on the PK and PD of cinacalcet in that cinacalcet exposure was greater in the non-fasting group. Therefore, cinacalcet may be better absorbed when taken with or immediately after eating.

\section{Acknowledgments}

The authors would like to thank all subjects for their participation in this study. We also would like to thank the clinical investigators, nurses, and study coordinators for their contributions to this study.

Funding: This project was supported by grants from the National Natural Sciences Foundation of China (81673515, 81870436, 81503160), Natural Science Foundation of Jiangsu Province (BK20161591), Six Talent Peaks Project in Jiangsu Province (2014-YY-001), Jiangsu Provincial Medical Youth Talent (QNRC2016215), Suzhou Science and Education Youth Project (KJXW2016067), Suzhou Industrial Technology Innovation (SYSD2016046), National Key Research \& Development Plan of the Ministry of Science and Technology of the People's Republic of China (2018YFC1314900, 2018YFC1314901), the 2016 Industry Prospecting and Common Key Technology Key Projects of Jiangsu Province Science and Technology Department (BE2016002-4), the 2017 Projects of Jiangsu Provincial Department of Finance (2150510), and the 2016 Projects of Nanjing Science Bureau (201608003).

\section{Footnote}

Reporting Checklist: The authors have completed the CONSORT reporting checklist. Available at http://dx.doi. org/10.21037/atm-20-1329

Peer Review File: Available at http://dx.doi.org/10.21037/ atm-20-1329

Data Sharing Statement: Available at http://dx.doi. org/10.21037/atm-20-1329

Conflicts of Interest: All authors have completed the ICMJE uniform disclosure form (available at http://dx.doi. org/10.21037/atm-20-1329). The authors have no conflicts of interest to declare.

Ethical Statement: The authors are accountable for all aspects of the work in ensuring that questions related to the accuracy or integrity of any part of the work are appropriately investigated and resolved. The study was conducted in accordance with the Declaration of Helsinki (as revised in 2013). The study was approved by ethics board of the First Affiliated Hospital of Nanjing Medical University (No. 2016-MD-208) and informed consent was taken from 


\section{Page 12 of 13}

all the patients.

Open Access Statement: This is an Open Access article distributed in accordance with the Creative Commons Attribution-NonCommercial-NoDerivs 4.0 International License (CC BY-NC-ND 4.0), which permits the noncommercial replication and distribution of the article with the strict proviso that no changes or edits are made and the original work is properly cited (including links to both the formal publication through the relevant DOI and the license). See: https://creativecommons.org/licenses/by-nc-nd/4.0/.

\section{References}

1. Takada D, Tsukamoto T, Fuse M, et al. The use of cinacalcet hinders the diagnosis of parathyroid carcinoma in a chronic dialysis patient: a case report. BMC Nephrol 2017; 18:315.

2. Ho LY, Wong PN, Sin HK, et al. Risk factors and clinical course of hungry bone syndrome after total parathyroidectomy in dialysis patients with secondary hyperparathyroidism. BMC Nephrol 2017;18:12.

3. Cunningham J, Locatelli F, Rodriguez M. Secondary hyperparathyroidism: pathogenesis, disease progression, and therapeutic options. Clin J Am Soc Nephrol 2011;6:913-21.

4. Sohn WY, Portale AA, Salusky IB, et al. An open-label, single-dose study to evaluate the safety, tolerability, pharmacokinetics, and pharmacodynamics of cinacalcet in pediatric subjects aged 28 days to $<6$ years with chronic kidney disease receiving dialysis. Pediatr Nephrol 2019;34:145-54.

5. Purrunsing Y, Zhang J, Cui Y, et al. Sixty-Two-Year-Old Male Suffering From Uremic Leontiasis Ossea Caused by Severe Secondary Hyperparathyroidism. JBMR Plus 2018;2:240-5.

6. Reiss AB, Miyawaki N, Moon J, et al. CKD, arterial calcification, atherosclerosis and bone health: Interrelationships and controversies. Atherosclerosis 2018;278:49-59.

7. Zawierucha J, Malyszko J, Malyszko JS, et al. Treatment of secondary hyperparathyroidism with paricalcitol with or without cinacalcet in hemodialysis patients. Pol Arch Intern Med 2017;127:840-5.

8. Zavvos V, Fyssa L, Papasotiriou M, et al. Long-Term Use of Cinacalcet in Kidney Transplant Recipients With Hypercalcemic Secondary Hyperparathyroidism: A Single-Center Prospective Study. Exp Clin Transplant
2018;16:287-93.

9. Eidman KE, Wetmore JB. Treatment of secondary hyperparathyroidism: How do cinacalcet and etelcalcetide differ? Semin Dial 2018;31:440-4.

10. Stollenwerk B, Iannazzo S, Akehurst R, et al. A DecisionAnalytic Model to Assess the Cost-Effectiveness of Etelcalcetide vs. Cinacalcet. Pharmacoeconomics 2018;36:603-12.

11. Yousaf F, Charytan C. Review of cinacalcet hydrochloride in the management of secondary hyperparathyroidism. Ren Fail 2014;36:131-8.

12. Dulfer RR, Franssen GJH, Hesselink DA, et al. Systematic review of surgical and medical treatment for tertiary hyperparathyroidism. Br J Surg 2017;104:804-13.

13. Mei C, Chen N, Ding X, et al. Efficacy and safety of Cinacalcet on secondary hyperparathyroidism in Chinese chronic kidney disease patients receiving hemodialysis. Hemodial Int 2016;20:589-600.

14. Floege J, Kubo Y, Floege A, et al. The Effect of Cinacalcet on Calcific Uremic Arteriolopathy Events in Patients Receiving Hemodialysis: The EVOLVE Trial. Clin J Am Soc Nephrol 2015;10:800-7.

15. Dixon M, Luthra V, Todd C. Use of cinacalcet in lithium-induced hyperparathyroidism. BMJ Case Rep 2018;2018:bcr2018225154.

16. Jeong S, Kim IW, Oh KH, et al. Pharmacogenetic analysis of cinacalcet response in secondary hyperparathyroidism patients. Drug Des Devel Ther 2016;10:2211-25.

17. Brunelli SM, Dluzniewski PJ, Cooper K, et al. Management of serum calcium reductions among patients on hemodialysis following cinacalcet initiation. Pharmacoepidemiol Drug Saf 2015;24:1058-67.

18. Lau WL, Obi Y, Kalantar-Zadeh K. Parathyroidectomy in the Management of Secondary Hyperparathyroidism. Clin J Am Soc Nephrol 2018;13:952-61.

19. Evenepoel P, Shroff R. Facing cinacalcet-induced hypocalcemia: sit back and relax? Kidney Int 2018;93:1275-7.

20. Schappacher-Tilp G, Fuertinger DH, Kotanko P. A MultiCompartment Model Capturing the Pharmacokinetics of the Calcimimetic Cinacalcet. Cell Physiol Biochem 2019;53:429-38.

21. Harris RZ, Salfi M, Sullivan JT, et al. Pharmacokinetics of cinacalcet hydrochloride when administered with ketoconazole. Clin Pharmacokinet 2007;46:495-501.

22. Padhi D, Harris R. Clinical pharmacokinetic and pharmacodynamic profile of cinacalcet hydrochloride. Clin Pharmacokinet 2009;48:303-11. 
23. Harris RZ, Salfi M, Posvar E, et al. Pharmacokinetics of desipramine $\mathrm{HCl}$ when administered with cinacalcet $\mathrm{HCl}$. Eur J Clin Pharmacol 2007;63:159-63.

24. Grzegorzewska AE, Paciorkowski M, Mostowska A, et al. Associations of the calcium-sensing receptor gene CASR rs7652589 SNP with nephrolithiasis and secondary hyperparathyroidism in haemodialysis patients. Sci Rep 2016;6:35188.

25. Kapur K, Johnson T, Beckmann ND, et al. Genome-wide meta-analysis for serum calcium identifies significantly associated SNPs near the calcium-sensing receptor (CASR) gene. PLoS Genet 2010;6:e1001035.

26. Wang LY, Zhang P, Wang HF, et al. Association of vitamin $\mathrm{D}$ receptor gene polymorphisms with end-stage renal disease and the development of high-turnover renal osteodystrophy in a Chinese population. Genet Mol Res 2016. doi: 10.4238/gmr.15026825.

27. Saarnio E, Pekkinen M, Itkonen ST, et al. Serum parathyroid hormone is related to genetic variation in vitamin $\mathrm{D}$ binding protein with respect to total, free, and bioavailable 25-hydroxyvitamin D in middle-aged Caucasians - a cross-sectional study. BMC Nutrition 2016;2:46.

28. Bai X, Xie J, Sun S, et al. The associations of genetic polymorphisms in CYP1A2 and CYP3A4 with clinical outcomes of breast cancer patients in northern China. Oncotarget 2017;8:38367-77.

29. Viikki M, Kampman O, Seppala N, et al. CYP1A2 polymorphism -1545C > T (rs2470890) is associated with increased side effects to clozapine. BMC Psychiatry 2014;14:50.

30. Lopez-Garcia MA, Feria-Romero IA, Serrano H, et al. Influence of genetic variants of CYP2D6, CYP2C9, CYP2C19 and CYP3A4 on antiepileptic drug metabolism in pediatric patients with refractory epilepsy. Pharmacol Rep 2017;69:504-11.

31. Sun B, Guo Y, Gao J, et al. Influence of CYP3A and ABCB1 polymorphisms on cyclosporine concentrations in renal transplant recipients. Pharmacogenomics

Cite this article as: Liu YJ, Sun LN, Cheng ZP, Qian Y, Ma ZQ, Zhang XH, Zhang HW, Xie LJ, Yu L, Yuan ZQY, Liu Y, Wang YQ. Pharmacogenetic and safety analysis of cinacalcet hydrochloride in healthy Chinese subjects. Ann Transl Med 2020;8(21):1385. doi: 10.21037/atm-20-1329
2017;18:1503-13.

32. Kawata T, Tokunaga S, Murai M, et al. A novel calcimimetic agent, evocalcet (MT-4580/KHK7580), suppresses the parathyroid cell function with little effect on the gastrointestinal tract or CYP isozymes in vivo and in vitro. PLoS One 2018;13:e0195316.

33. Hu C, Hu X, Wang C, et al. Bioequivalence and Pharmacokinetics of Bisoprolol-Amlodipine $5 \mathrm{mg} / 5 \mathrm{mg}$ Combination Tablet versus Bisoprolol $5 \mathrm{mg}$ Tablet and Amlodipine $5 \mathrm{mg}$ Tablet: An Open-Label, Randomized, Two-Sequence Crossover Study in Healthy Chinese Subjects. Clin Drug Investig 2018;38:1145-54.

34. Marchini J, Howie B, Myers S, et al. A new multipoint method for genome-wide association studies by imputation of genotypes. Nat Genet 2007;39:906-13.

35. Komaba H, Kakuta T, Fukagawa M. Management of secondary hyperparathyroidism: how and why? Clin Exp Nephrol 2017;21:37-45.

36. Greeviroj P, Kitrungphaiboon T, Katavetin P, et al. Cinacalcet for Treatment of Chronic Kidney Disease-Mineral and Bone Disorder: A Meta-Analysis of Randomized Controlled Trials. Nephron 2018;139:197-210.

37. Liu H, Wang H, Liu T, et al. Pharmacokinetic and Pharmacodynamic Properties of Cinacalcet (KRN1493) in Chinese Healthy Volunteers: A Randomized, Open-label, Single Ascending-dose and Multiple-dose, Parallel-group Study. Clin Ther 2016;38:348-57.

38. Cruzado JM, Moreno P, Torregrosa JV, et al. A Randomized Study Comparing Parathyroidectomy with Cinacalcet for Treating Hypercalcemia in Kidney Allograft Recipients with Hyperparathyroidism. J Am Soc Nephrol 2016;27:2487-94.

39. Drüeke TB. Cinacalcet treatment in dialysis patients with secondary hyperparathyroidism: effects and open issues. Ther Apher Dial 2008;12 Suppl 1:S2-12.

40. Cinacalcet: new drug. Secondary hyperparathyroidism: where are the clinical data? Prescrire Int 2006;15:90-3.

(English Language Editors: B. Madden and J. Chapnick) 